\title{
Liquid Nitrogen-Cooled Fourier Transform Spectrometer System for Measuring Atmospheric Emission at High Altitudes
}

\author{
Frank H. Murcray, Frank J. Murcray and David G. Murcray \\ University of Denver, Denver, CO 80208
}

JAMES PRITCHARD

Idealab, Franklin, MA 02038

GEORGE VANASSE

Air Force Geophysics Laboratory, Bedford, MA 01730

HAJIME SAKaI

University of Massachusetts, Amherst, MA 01002

(Manuscript received 30 March 1984, in final form 27 August 1984)

\section{ABSTRACT}

\begin{abstract}
A cryogenically cooled FTS system capable of a spectral resolution of $0.06 \mathrm{~cm}^{-1}$ is described. The entire interferometer is operated at $\mathbf{L N}_{2}$ temperature with the exception of the Ge:Cu detector, which is cooled to below $10 \mathrm{~K}$. In order to maintain alignment while the unit is cold, a cat's eye optical system was employed, and all mechanical components fabricated from the same stress-relieved steel.

The system was constructed to measure atmospheric emission spectra from a balloon platform. The requirements on the electronics and data handling systems imposed by the remote operation and balloon environment are discussed.

Some data obtained on a balloon flight launched on 23 October 1983 are presented. These show that the instrument has the sensitivity to measure the atmospheric emission at high altitudes with the spectral resolution desired $\left(0.06 \mathrm{~cm}^{-1}\right)$.
\end{abstract}

\section{Introduction}

The absorption and emission of electromagnetic radiation by the earth and atmosphere is of interest in a number of disciplines. Solar energy, primarily as visible wavelength radiation, does not interact strongly with a clear atmosphere, but after absorption at the earth's surface it is eventually reradiated at infrared wavelengths. Many of these wavelengths react strongly with atmospheric gases. As a result, the emission and absorption of infrared radiation by the earth's atmosphere is a major factor in both long-term global climatic effects and more local shorter-term meteorological phenomena.

Elsasser (1942), in one of the initial attempts to treat radiative transfer on a quantitative basis, pointed out that high spectral resolution was not really desirable in experimental studies because the theoretical treatment could not handle the detail present in atmospheric spectra. The situation has changed over the years and with the development of large comput- ers, it is now possible to treat the problem in great spectral detail. At the same time, it has become apparent that spectral detail is required for calculations of the energy exchange to the accuracy necessary for assessment of the climatic impact resulting from increased atmospheric concentrations of radiatively important compounds $\left(\mathrm{CO}_{2}, \mathrm{~N}_{2} \mathrm{O}, \mathrm{CH}_{4}\right.$, etc.). The inversion process, where temperature profiles are calculated from spectral measurements of the $\mathrm{CO}_{2}$ emissions, also increases in absolute accuracy with the spectral resolution employed (Smith et al., 1983).

Infrared techniques are also being used to measure the atmospheric concentration of a number of chemical species. In this case, spectral resolution is important in separating the spectral features of interest from those of interfering species. Thus, both from the standpoint of testing radiative transfer calculations and for the measurement of trace constituents, atmospheric emission measurements are being pushed to higher resolution.

Various instruments including prism spectrometers, 
circular variable filters, grating spectrometers and Fourier transform spectrometer systems have been used to measure atmospheric emissions on a spectral basis. The spectral resolution utilized in most of these measurements was limited by the need for high throughput to achieve the required signal to noise. For warm instruments, the Fourier transform spectrometer, while presenting severe technical problems, had a clear theoretical advantage both in having high throughput and of measuring the power in all frequencies simultaneously (i.e., the multiplex or Fellgett advantage).

A fundamental limitation on the sensitivity of all these systems is the noise generated by statistical fluctuations in the photon flux on the detector. When the photon flux from the source is very low, as in the case of atmospheric emission at high altitude, "background" photons emitted by the optics and detector surroundings are the dominant noise source, and dramatic improvements in sensitivity can be achieved by reducing this background flux. This led to the design of various cryogenically cooled spectrometer systems, including small grating spectrometers (Murcray et al., 1975), circular variable filters (Wyatt, 1975 ) and low-resolution Fourier transform systems (Murcray, 1978). In this cold optics regime, the choice of instruments is less straightforward since the multiplex advantage of the interferometer is lost when the fluctuations in the source photons become the dominant source of noise (Decker, 1977). The throughput advantage can be partially compensated for by the larger optics, which are practical for the grating system, and is a small factor in the case of the circular variable filter. The choice of spectrometer type for low to moderate resolution measurements is therefore likely to be made on other grounds such as ease of operation or data handling requirements.

Liquid helium cooled grating spectrometers have been used for midinfrared stratospheric measurements since the mid 1970s (Murcray et al., 1975). These instruments have been successful in measuring very low levels of emission at a resolution better than 0.5 $\mathrm{cm}^{-1}$. As noted above, an increase in resolution would be advantageous for many studies. An increase in resolution by a factor of 5 , while maintaining comparable sensitivity, would require a major increase in the physical size of a grating spectrometer. The Fourier transform instrument needs only $5 \mathrm{~cm}$ of travel (10 cm optical path difference) to achieve 0.1 $\mathrm{cm}^{-1}$ resolution and, hence, can be constructed to physical dimensions comparable with those of lower resolution systems. In addition, cooling to below 100 $\mathrm{K}$ is sufficient for reducing the noise from background flux well below that of the source photons in most applications. A cryogenic Fourier transform system was therefore selected as a means of obtaining the high-resolution data desired. A block diagram of the system is shown in Fig. 1.

\section{Interferometer system}

Maintaining optical alignment of a moving mirror interferometer system is not easy even for normal operating conditions. Aligning and maintaining alignment of such a system when it is cooled to $80 \mathrm{~K}$ and not accessible for adjustment is a major problem, and one that has received attention for over a decade. Various approaches have been used in attempting to overcome this problem. The one employed in this unit was developed by J. Pritchard of Idealab. The approach is to reduce, insofar as is possible, the dependence of the alignment on temperature through several design and fabrication techniques:

1) All mechanical components were constructed of the same material, a number 2 steel, and all units went through numerous annealing cycles to reduce thermal stress.

2) The entire interferorneter was assembled on a solid base plate of the same material.

3) A cat's eye retroreflecting optical system (shown schematically in Fig. 2) was chosen as providing automatic compensation for slight rotations of the interferometer arms.

4) The ways that support the movable carriage must provide a smooth motion at $80 \mathrm{~K}$. Ball bearing slides together with a dry lubricant were found to satisfy the mechanical requirements even in these severe temperature conditions. The moving mirror is driven by a speaker-coil magnetic motor and its position monitored by both a mechanical transducer and fringes formed by a cw $\mathrm{He}-\mathrm{Ne}$ laser beam. The beamsplitter is $\mathrm{KCl}$ coated with $\mathrm{Ge}$ for maximum efficiency in the $8-13 \mu \mathrm{m}$ region. It is paired with a compensator plate of similar optical path. They are jointly mounted to a supporting frame using a springloaded retaining ring, which serves as a cushion for the thermal stress associated with the temperature cycle. A Ge:Cu photoconductor is used as the detector of the infrared radiation. This detector has to be cooled to below $10 \mathrm{~K}$ to obtain any sensitivity, hence, provision had to be made for a second Dewar to hold liquid helium for cooling the detector. The detector and Dewar also mount on the optical base plate as shown in Fig. 3.

The interferometer mounting plate is suspended in a vacuum cryostat by means of four fiberglass reinforced epoxy pillars. The plate is in thermal contact with a 30 liter liquid nitrogen reservoir. Once the instrument is cold, this reservoir provides sufficient cooling capacity to keep the instrument below $90 \mathrm{~K}$ for at least 20 hours. Vacuum seal is provided by the final end plates. These plates are equipped with a $\mathrm{ZnSe}$ window on the front plate and suitable cryogen fill tubes on the back plate. The window can be cooled to $80 \mathrm{~K}$ (for the 23 October 1983 flight, the window operated near ambient). 


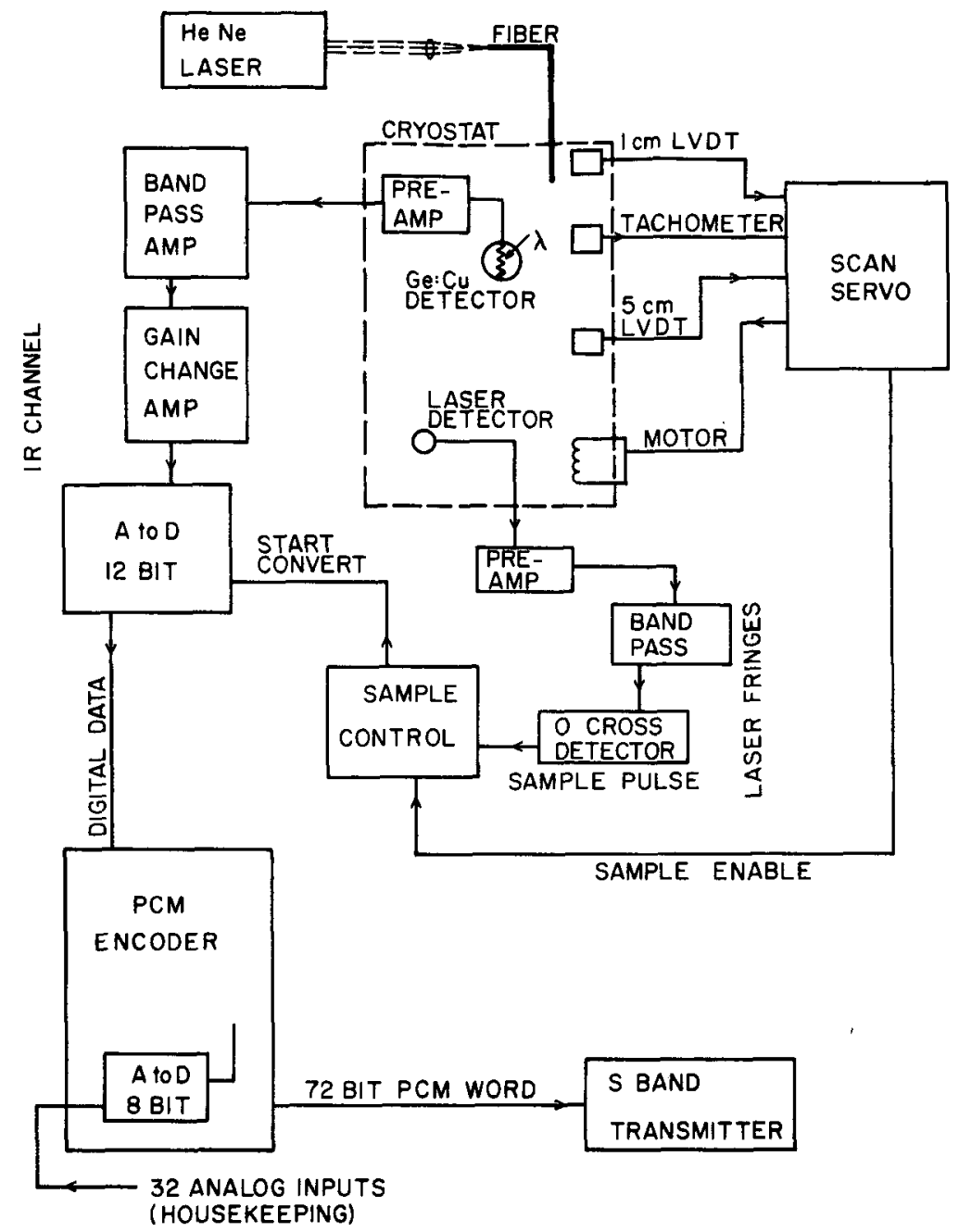

FIG. 1. A schematic diagram showing the functional relationships between the various electro-optic, electromechanical and electronic systems of the interferometer. The LVDTs (linear variable differential transformers) are transducers that sense the position of the moving cat's eye.

It is very important that the interferogram be sampled at precise increments of the path difference in the interferometer. A He-Ne laser beam traversing the interferometer system produces interference fringes that provide a very accurate measure of the change in pathlength and, therefore, a precise means of sampling the interferograms at the proper points. The implementation of this scheme is complicated in a cryogenic instrument, since the normal $\mathrm{HeNe}$ tube will not operate at $\mathrm{LN}_{2}$ temperature. Operation of the tube within the cryostat would require that the laser be thermally isolated from the instrument and, at the same time, mechanically coupled to it rigidly enough to maintain optical alignment. This had been done on previous cryogenic units, but many problems were encountered. Since the instrument baseplate is thermally isolated from the cryostat, relative move- ment between them, particularly during cooling cycles, was likely. Therefore, it was not possible to operate the laser outside the cryostat and simply direct the beam into the interferometer. Instead, the laser energy was coupled into an optical fiber whose output end was clamped to the interferometer baseplate. The contrast of the fringes produced by this system proved to be very sensitive to slight variations in fiber temperature and to the launch of the radiation into the fiber, apparently a result of changing propagation paths in the fiber and the particular characteristics of the cat's eye optics. A changeover to a single-mode type fiber and provisions to reduce the temperature variation of the portions of the fiber external to the cryostat reduced the variation to the point that the system operated well in the balloon flight environment. 


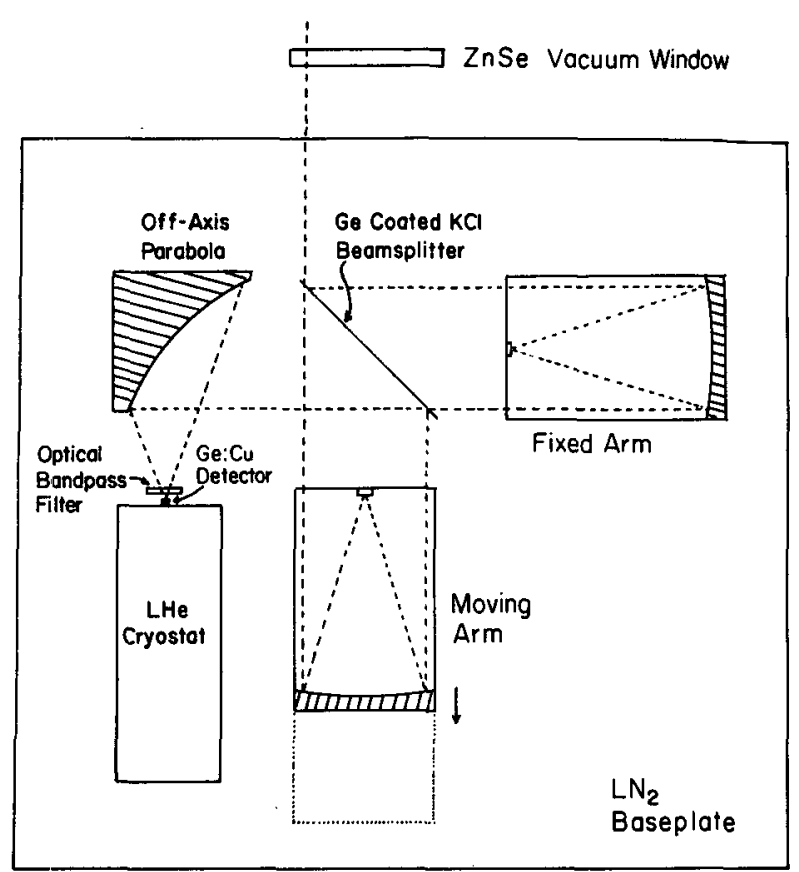

FIG. 2. Schematic of the interferometer optical system. In the interest of clarity, only one marginal ray is depicted.

\section{Electrical systems}

The instrument interior to the cryostat operates in a vacuum and, once cold, is in a very stable temperature environment. The electronics and other systems external to the cryostat are subject to rapid and rather extreme variation in ambient temperature and pressure in the course of a balloon flight, and all systems must function without intervention (except for a few radio-transmitted commands). Primary design emphasis was therefore placed on reliability rather than on the versatility desirable in a laboratory system.

All electronics with the exception of DC-to-DC converters are insulated and provided with thermostatic heaters. The He-Ne laser tube and associated high-voltage supply are housed in a pressurized aluminum cylinder to prevent arc-over at the high altitudes. Laser radiation exits the enclosure through a $5 \mathrm{~cm}$ diameter quartz window.

\section{Mirror drive servo}

The system is designed to produce a single-sided interferogram, i.e., designating the optical paths through the fixed and moving arms as $L_{f}$ and $L_{m}$, respectively; the optical path difference $L_{m}-L_{f}$ is varied from -0.1 to $+10 \mathrm{~cm}$. This provides sufficient data at negative (as defined here) path differences for phase corrections and nearly doubles the resolution achieved by the $5 \mathrm{~cm}$ mechanical motion of the mirror.

As indicated above, the moving cat's eye was driven by a speaker coil arrangement powered by complementary power transistors driven in turn by an operational amplifier. The error signal for the drive is obtained by comparing the output of a linear tachometer against a preset value. This velocity servo, in addition to its inherent stability, has a virtue (important in balloon operation) of limiting the accelerations resulting from such perturbations as unattended powerups and launch shocks. The position of the moving mirror is sensed by a $5 \mathrm{~cm}$ linear variable differential transformer (LVDT), and the turnaround at maximum path difference is initiated by this sensor.

The cat's eye is returned to zero path difference at five times the velocity of the outward scan. Data taking is suspended during the return, avoiding several complications involved in data taking on reverse scans, at a sacrifice of not more than $20 \%$ of the observation time. Greater precision is required for the scan reversal near zero path difference (ZPD), so a separate $1 \mathrm{~cm}$ range LVDT is used to activate the turnaround. The long-term stability of this turnaround

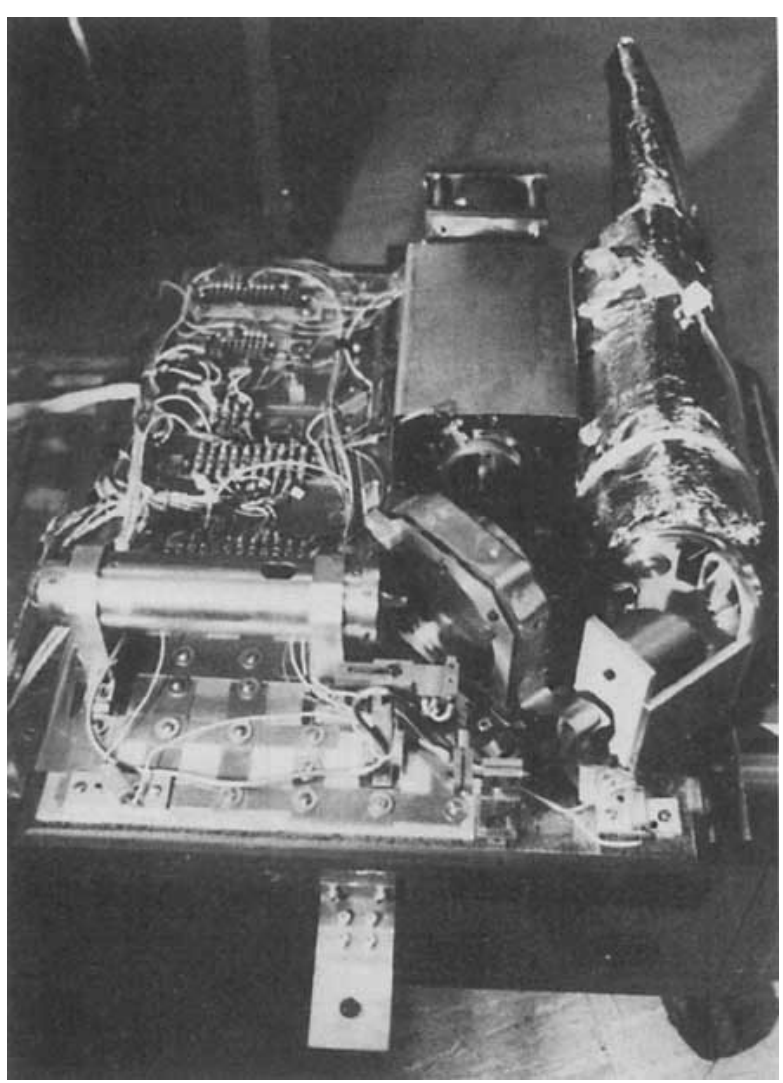

FiG. 3. The interferometer as prepared for installation in the cryostat. The $\mathrm{KCl}$ beamsplitter is in the center foreground with the fixed cat's eye extending to the left. An oxygen-free high conductivity copper shield covers the traveling cat's eye assembly to the rear. On the right are the detector collecting optics and the LHe reservoir. Copper strapping on the base plate and components improves the thermal contact with the $\mathrm{LN}_{2}$ reservoir. 
position is of the order of $10 \mu \mathrm{m}$ over the duration of a balloon flight.

This drive system has performed well since implemented, although the tachometer output is at quite a low level and requires care to avoid picking up extraneous signals in the high-gain amplifier necessary to utilize this signal.

\section{Main data channel}

The interferogram information is represented by photon-induced currents in the Ge:Cu detector. These are converted to signal voltages by an Analog Devices $515 \mathrm{~K}$ operational amplifier operated as a current-tovoltage converter. This circuit has the advantage of better than $1 \%$ linearity over a detector impedance range of $10^{6}-11^{11} \Omega$ and low effective input capacitance. The operational amplifier is mounted in a thermal enclosure a few centimeters from the detector. Connection of the detector to the summing input of the operational amplifier is through a shielded number 36 manganin wire. The shield is connected to the noninverting input and to case ground. This configuration minimizes microphonics and extraneous pickup. Some of these devices exhibit permanent degradation in performance after exposure to cryogenic temperatures, so the device is maintained above $220 \mathrm{~K}$ whenever the cryostat is cold or cooling. The feedback resistor $\left(10^{8}-10^{9} \mathrm{ohms}\right)$ is mounted to the helium heat sink to minimize the Johnson noise from this source. The preamplifier output is brought out of the cryostat where it is routed to a DC impedance monitor and to interstage electronics for further amplification and filtering.

The frequency range of the interferogram is determined by the optical bandpass of the filter at the detector and the mirror velocity. Active filters limit the electrical bandpass to a similar range but are set somewhat broader to avoid excessive phase shifts for information near the optical frequency cutoffs.

On a typical balloon flight the instrument will measure radiances from sources that vary by orders of magnitude in strength and spectral distribution. The output signal is maintained near the optimum level for digitization by monitoring the peak amplitude of the interferogram and incrementing or decrementing the gain of the output stage by factors of 2 if the peak value lies outside the optimum range. The amplitude of the ZPD peak for a single interferogram may exceed the amplitude at the end of the scan by several orders of magnitude, depending on the spectral content. This can exceed the dynamic range of the 12-bit analog-to-digital converter and lead to digitization noise in the interferogram. This is avoided by increasing the gain once the ZPD region has been scanned. Typically, this positional gain change is activated at a path difference of $0.25-0.50 \mathrm{~mm}$ from ZPD. The output of the final stage is fed to the input of the A-to-D converter where it is converted to a 12-bit binary number upon command from the sample control.

\section{Sampling control}

The interferogram must be measured at precise equal increments of the optical path difference. The fringes, produced in a $\mathrm{He}-\mathrm{Ne}$ laser beam which traverses a small portion of the beamsplitter (coated for visible wavelength efficiency), provide an accurate monitor of the mirror motion. The laser channel output is focused on a silicon PIN photodiode, which is operated unbiased. The diode output is brought external to the cryostat to the summing input of a $515 \mathrm{~K}$ operational amplifier whose output goes directly to an intensity monitor and also to a narrow-band high-gain amplifier. The high-amplitude sine wave generated by this amplifier is converted to two pulses per cycle by a zero-crossing detector. These pulses are routed to counters where the sample spacing in multiples of $0.3164 \mu \mathrm{m}$ can be selected by manual switches. The output pulses at the spacing selected initiate the conversion cycle in the A-to-D converter. With the wide optical bandpass employed so far, a $0.6328 \mu \mathrm{m}$ sample spacing has been used.

\section{Scan sequence}

The active filters which set the bandpasses of the infrared and laser channels are constructed from fixed components. The scan velocity, although adjustable over a wide range, must be compatible with these bandpasses and is therefore fixed once they are determined. A 30-second scan sequence has been selected as most compatible with drive characteristics, data rates and experiment parameters.

A scan sequence is initiated by a pulse derived from the $1 \mathrm{~cm}$ LVDT at the zero path difference end of the scan. This start of scan pulse sets the velocity reference polarity to forward and initiates the laser fringe counter. When the fringe count reaches a value selected by manually set switches, sample pulses are initiated. The switches are set to initiate sampling about 400 samples before ZPD. This provides adequate data for phase correction of the interferogram. A sample-pulse counter is initiated at the start of sampling; when this count reaches a set value (again selectable by manual switches), the positional gain change is activated (normally at about 400 points beyond ZPD, i.e., 800 points from the start of sampling). The $5 \mathrm{~cm}$ LVDT monitors the position of the mirror carriage, and a pulse derived from this transducer terminates the scan at the length selected. The end of scan pulse sets the tachometer reference to the reverse polarity, shuts off sampling pulses and resets all counters to zero. The return velocity is independently variable and is set much higher than the forward velocity so that of the 30 -second cycle, about 25 seconds are devoted to the forward scan and 5 seconds to the reverse. The cycle is repeated automatically whenever the drive circuitry is powered. 


\section{Auxiliary systems}

The value of the atmospheric emission measurements is enhanced if they can be made at several elevation angles from just above the earth's limb to several degrees above the balloon horizontal. At the higher elevation angles, the atmospheric emissions are low, ànd emissions from an external mirror would be a significant part of the measurement. It is desirable therefore to change the line of sight by rotating the instrument rather than by injecting a mirror into the field of view.

Although this is a somewhat formidable task when it involves a 1300 pound cryostat, it is accomplished (albeit slowly) by a small DC motor driving a screwtype jack. The instrument is launched at an elevation angle of $4.7^{\circ}$ and lowered by discrete steps (controlled by microswitch indents) through radio commands from the ground station. When the minimum angle $-5.4^{\circ}$ is reached, a single repetition of the command returns the instrument to the maximum elevation indent.

Nadir or near-nadir viewing results in complex spectra directly applicable to radiation balance calculations, but it cannot be accomplished by rotating the instrument. The signal levels in this case are comparatively high so that a plane mirror oriented $45^{\circ}$ from the horizontal can be injected into the field of view for this measurement without introducing appreciable error. The mirror is injected by a rack and pinion assembly, again powered by a small DC motor. Injection is initiated by command from the ground, and the motor is stopped at the proper position by a microswitch indent. A repetition of the command injects a blackbody calibration source. The next command returns all elements to their original position, leaving the field of view unobstructed.

\section{Telemetry}

The interferogram digital word along with a variety of monitor and housekeeping functions must be transmitted via an S-band telemetry link for recording at the ground station. Therefore, the analog signals must be converted to digital form and combined with the interferogram word into a 72-bit PCM frame. This function is performed by a custom encoder acquired by AFGL. Optimum signal recovery from the PCM data stream requires a constant bit/frame rate and, hence, cannot be synchronized with interferogram sampling. It is imperative, however, that no samples be missed or repeated in the transmitted data. The PCM frame rate is high enough to insure that each interferogram sample will be read-in at least once. A pulse from the encoder signifying that the data have been read-in clears the A-to-D outputs and resets a data-valid bit. Additional frames transmitted before a new interferogram sample is ready contain empty interferogram words.

\section{Auxiliary data}

The PCM encoder generates a 72-bit frame. Of the 72 bits, 40 are devoted to the sync-word, interferogram word and subframe ID, and the remaining 32 bits are devoted to auxiliary data. Frames are generated at a rate of $10^{4} \mathrm{~s}^{-1}$, a much higher rate than required for these auxiliary measurements. Auxiliary inputs are therefore commutated, permitting 32 information channels to be sampled at a rate of 200 samples per second each. These channels include various instrument functions including the IR detector impedance, laser intensity, laser fringe amplitude, drive position, tachometer output, amplifier gain setting and various internal temperatures. Additional inputs give altitude (pressure), gondola azimuth, instrument elevation and various temperatures external to the cryostat including that of the calibration source. Balloon control functions are also included in these channels.

\section{Power}

The primary power sources for the balloon payload are nominal $28 \mathrm{~V}$ silver-zinc battery packs. A $70 \mathrm{~A}$ $\mathrm{h}^{-1}$ pack powers all interferometer systems except the balloon control package and PCM telemetry. Regulated $+15 \mathrm{~V}$ power for analog electronics and $+5 \mathrm{~V}$ power for the digital functions is supplied by switching DC-to-DC converters with the exception of the bias and preamplifier supplies for the infrared channel, which are derived from alkaline cells. The laser power supply, relay functions and thermostated heaters for the electronic enclosures are supplied directly from the battery.

\section{Ground recording}

The PCM bit stream at the output of the ground station S-band receiver is routed through a bit synchronizer to generate sharp clean transitions for recording directly onto magnetic tape. A parallel bit synchronizer output is fed through an interface to a Nova 1200 minicomputer. The computer decodes the PCM word and, after discarding the sync-frames, empty frames and redundant samples of analog data, reformats the remainder and records it on magnetic tape. Since recovery of a full-resolution spectrum requires calculation of the Fourier transform for a 160000 point interferogram, these computations are deferred until after the flight. (The reader is referred to works such as Vanasse, 1977, for the details of such computations.) However, portions of the current interferogram can be displayed on a storage oscilloscope for real time monitoring of the instrument performance.

\section{Results}

The instrument was flown from Hollomon AFB on 23 October 1983. For this flight a $142000 \mathrm{M}^{3}$ 


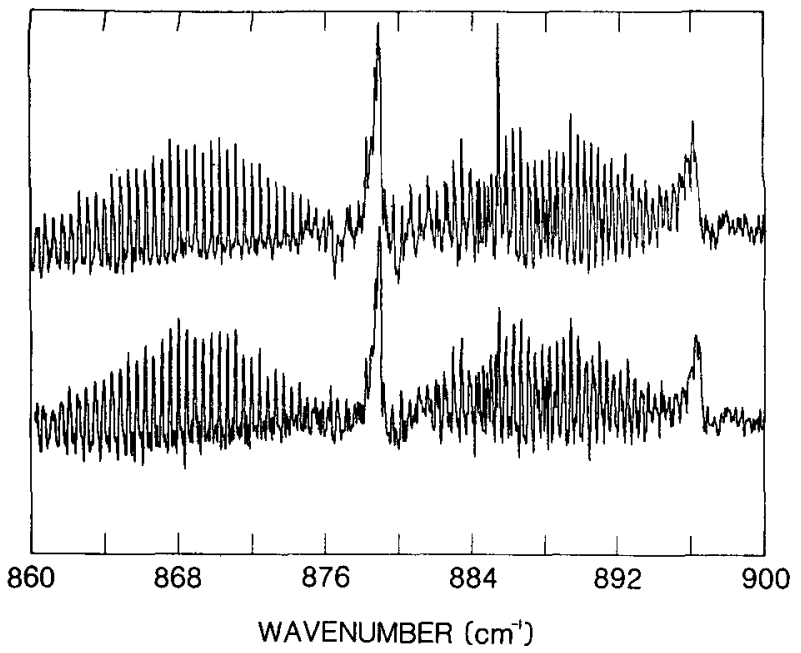

FIG. 4. Comparison of the atmospheric emission spectrum in the $860-900 \mathrm{~cm}^{-1}$ region as measured from $30 \mathrm{~km}$ altitude at an elevation angle of $-2.9^{\circ}$ (bottom), with a $0.06 \mathrm{~cm}^{-1}$ resolution absorption spectrum of $\mathrm{HNO}_{3}$ (top). The absorption spectrum was measured with $2.2 \mathrm{~mm} \mathrm{Hg}$ pressure of $\mathrm{HNO}_{3}$ in a $5 \mathrm{~cm}$ cell. A Nernst glower at $1600 \mathrm{~K}$ was used as a source for the absorption measurement.

balloon was used. The fully loaded gondola weighed $1140 \mathrm{~kg}$. In addition, $200 \mathrm{~kg}$ of pourable ballast were included for control. With this weight, the theoretical altitude for the flight was $30 \mathrm{~km}$. The balloon was launched at 0610 MDT and reached float altitude at 0800 . The system remained at float for about $30 \mathrm{~min}$ and then began a gradual descent apparently due to a leak in the balloon. A gradual descent was maintained by ballasting until 1015 MDT when the flight was terminated by command. At that time the balloon was at an altitude of $19 \mathrm{~km}$. The gondola impacted near Carlsbad, New Mexico and was recovered in good condition.

When the balloon reached float, the instrument was stepped to view an angle of $2.9^{\circ}$ below horizontal. Several scans were taken at this viewing angle, and then the instrument viewing angle was changed to $5.4^{\circ}$ below horizontal. At this time it became evident that the balloon was descending and the remainder of the flight was used to obtain calibration data and to measure the upwelling radiation by viewing the nadir.

Figure 4 shows a portion of the spectra obtained with the instrument viewing the earth's atmosphere $2.9^{\circ}$ below horizontal. Under this geometry, the system line of sight traverses the atmosphere at a tangent height of $21.6 \mathrm{~km}$. The dominant atmospheric emitter in the spectral region covered in Fig. 4 is $\mathrm{HNO}_{3}$. Also shown in the figure is a laboratory absorption spectrum obtained with a different Fourier transform spectrometer system with a theoretical resolution of $0.06 \mathrm{~cm}^{-1}$. For this measurement, a 5 $\mathrm{cm}$ absorption cell was used with $\mathrm{HNO}_{3}$ at a pressure of $2.2 \mathrm{~mm}$ and a temperature of $296 \mathrm{~K}$. Examination of the figure verifies that the atmospheric emission is predominantly due to $\mathrm{HNO}_{3}$. It also shows that the resolution achieved with the balloon-borne interferometer is comparable to that achieved with the laboratory interferometer. In comparing the spectra it should be kept in mind that the flight spectrum is an emission spectrum of $\mathrm{HNO}_{3}$ at a temperature of $250 \mathrm{~K}$ and that it may also include features due to constituents other than $\mathrm{HNO}_{3}$. The spectral region presented here lies in the middle of the so-called "atmospheric window" between 10 and $12 \mu \mathrm{m}$ and thus represents a region of minimum spectral radiance. The spectra also demonstrates that the system not only achieved the desired resolution but has sufficient sensitivity to observe atmospheric emission at high altitudes at that resolution.

Acknowledgments. The balloon operations were handled by AFGL personnel under the direction of Arthur Giannetti. The cooperative attitude and competence of this group contributed much to the success of the experiment as well as the balloon operations. All those who contributed cannot be acknowledged, but S.Sgt G. Clements and M.Sgt S. Longshore must be mentioned as well as Sgt. T. Dyer. This work was supported by the AFOSR unoier Atmospheric Science Project 2310 and performed as part of AFGL Task 2310G1. This paper has been approved for public release under ESD 84225 dated 20 March 1984.

\section{REFERENCES}

Decker, J. A.; Jr., 1977: Hadamord-transform spectrometry. Spectrometric Techniques, Vol. 1, G. A. Vanasse, Ed., Academic Press, 209-212.

Elsasser, W. M., 1942: Heat transfer by infrared radiation in the atmosphere. Harvard Meteorological Studies, No. 6, 107 pp.

Murcray, D. G., 1978: Infrared spectroscopic instrumentation for stratospheric research. National Center for Atmospheric Research, Atmos. Technol., 9, 73-79.

- D. B. Barker, J.' N. Brooks, A. Goldman and W. J. Williams, 1975: Seasonal and latitudinal variation of the stratospheric concentration of $\mathrm{HNO}_{3}$. Geophys. Res. Lett., 2, 223-225.

Smith, W. L., H. E. Revercomb, H. B. Howell and H. M. Woolf, 1983: HIS, a satellite instrument to observe temperature and moisture profiles with high vertical resolution. Presented at the Fifth Annual Atmospheric Radiation Conf., Baltimore, Amer. Meteor. Soc., 1-10.

Vanasse, G. A., 1977: Spectrometric Techniques, Vol. 1. Academic Press, 355 pp.

Wyatt, C. L., 1975: Infrared spectrometer liquid helium cooled rocket borne circular variable filter. Appl. Opt., 14, 30863091 . 